\title{
A disposable DNA methylation sensor based on the printable graphene field effect transistor
}

\author{
Yunfang Jia ${ }^{1}{ }^{*}$, Jizhao Zhang ${ }^{1}$ and Qingjie Fan ${ }^{2}$ \\ ${ }^{1}$ College of Electronic Information and Optic Engineering, Nankai University, Tianjin, China \\ ${ }^{2}$ Lanbiao Electronic Technology Development Co., Ltd., Tianjin, China
}

\begin{abstract}
The detection of DNA methylation is necessary for the research of epigenetics. In this work we would like to propose a disposable DNA methylation sensor by using graphene field effect transistor (GFET) as the sensing platform. In this component, the liquid-phase exfoliated graphene (LEG) nanosheets were drop-coated on the flexible substrates of polyethylene terephthalate (PET) films. Then, the interdigital structured electrodes (named as source and drain) were printed on the LEG coated PET films to form the expected GFETs. Thirdly, the carbon dots (CDs) decoration was conducted and examined on the asprepared GFETs to evaluate the influence of CDs, as well as optimize CDs' concentration. At last, the immune identification-based sensing strategy was utilized on the CDs modified GFETs to develop the concerned DNA methylation sensor. The experimental data indicate the proposed sensors could be a potential experimental tool for epigenetic research.
\end{abstract}

\section{Introduction}

DNA methylation is a common epigenetic phenomenon, which occurs in cytosine (C). Under the action of methyltransferase, methyl is covalently bound to the fifth carbon atom of $\mathrm{CpG}$ dinucleotide cytosine to form 5methylcytosine $(5 \mathrm{mC})$ [1]. It has been believed an efficient method for the DNA methylation detection is of significance to understand the internal mechanism of gene expression, and improve the level of clinical screening, diagnosis, treatment, and prognosis of related diseases, so lots of efforts had been made in the disciplines of biology, chemistry and electronics $[2,3,4$, 5].

The emergence of graphene field effect transistor (GFET) brought vitalities to almost all kinds of biosensors due to graphene's high electron mobility and specific surface area $[6,7]$. In our previous works, we tried to exploit it as the sensors for DNA methylation for DNA extracts from tumor cells [3] and tumor tissues [5]. To exploit it as a low-cost and practicable device for the clinical and biological applications, we would like to utilize the printing technique, which is one of the most popular electronic fabrication technologies.

In this work, the polyethylene terephthalate (PET) films were used as the substrates, the graphene material used in GFET was the liquid exfoliated graphene (LEG) by using thee cosolvent of dimethyl-formamide (DMF) and water. The interdigital electrodes of source and drain were printed on the specified locations to form the basic devices of GFET. According to the previously experiments [5], the decoration of CDs was conducted to enhance the amount of single strand DNA (ssDNA) which were waiting to be tested. At last, the determination of the $5 \mathrm{mC}$ positions on the tested ssDNA are realized by using the similar immune strategy in our previous works $[3,5]$, i.e., the antibodies of $5 \mathrm{mC}$ (anti$5 \mathrm{mC}$ ) were incubated on the ssDNA anchored GFETs, the changed currents were recorded to evaluate the sensitivity for DNA methylation.

The experimental results demonstrated this PET supported GFETs can be utilized for the DNA methylation assay. The proposed method for fabricating GFET is a totally solution processable processes with the merits of easy-to-produce and high performance-price ratio, the prepared GFET based DNA methylation sensor is disposable which makes it as an appealing device for constructing an efficient DNA methylation detection platform.

\section{Experiments and methods}

\subsection{Materials}

The main chemicals for fabricating the LEG based GFET are outlined at here: (1) graphite powder $(99 \%$, 325 mesh) from Huawei Ruike Chemical Co., LTD, Beijing, China; (2) DMF from J\&K Scientific, USA; (3) glutaraldehyde (GA) and 3-amino-propyl-triethoxysilane (APTES, from Sigma-Aldrich, USA; (4) citric acid (CA) and ethylenediamine (EDA) from Tianjin chemical reagent Co., Tianjin, China. The first three of the chemicals were used to prepare LEG and make it coated on PET films (thickness is $0.25 \mathrm{~mm}$ ) which were purchased from Huaxun Plastic and Rubber Co., LTD,

\footnotetext{
* Corresponding author: jiayf@nankai.edu.cn
} 
Guangdong, China. The conductive paste and insulation ink were from Saiya electronic pulp Co., Ltd, Shenzhen, China. The third chemical was used to active the PET substrates and the coated LEG layer on them. The fourth chemicals were used for synthetizing the CDs material by using the hydrothermal method. The printed devices were encapsulated by Silicone rubber (NANDA 703) from Liyang Kangda New Material Co., Ltd., Jiangsu, China.

The sequences of the tested ssDNAs which were ordered by Saigon Biotech Co. Ltd., Shanghai, China, are listed in Table 1. They are identical, with the only difference in the amount of $5 \mathrm{mC}$ sites which are marked as the underlined $\mathrm{C}$ in Table 1.

Table 1. The sequences of the tested single strand DNA.

\begin{tabular}{|c|c|}
\hline $\begin{array}{l}\text { Name of } \\
\text { ssDNA }\end{array}$ & Sequences of the bases \\
\hline T0 & $\begin{array}{c}\text { 5'-TTG CGC GGC GTC CGT CCT } \\
\text { GTT GAC TTC-3' }\end{array}$ \\
\hline $\mathrm{T} 1$ & $\begin{array}{c}\text { 5'-TTG CGC GGC GTC CGT CCT } \\
\text { GTT GAC TTC-3' }\end{array}$ \\
\hline $\mathrm{T} 2$ & $\begin{array}{c}\text { 5'-TTG CGC GGC GTC CGT CCET } \\
\text { GTT GAC TTC-3' }\end{array}$ \\
\hline $\mathrm{T} 3$ & 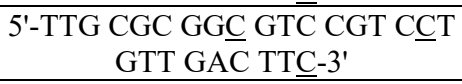 \\
\hline $\mathrm{T} 4$ & 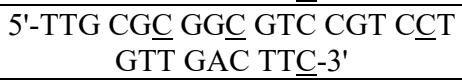 \\
\hline
\end{tabular}

\subsection{Apparatus}

High pressure reactor (CF-50, Gongyi Yuhua Instrument Co., Ltd.), magnetic stirrer (TDY-2, Beijing fangtongda Technology Co., Ltd.), ultrasonic cleaner (GT sonic-p20, Guangdong Gute ultrasonic Co., Ltd.), centrifuge (TGL50ws, Jintan Tianjing experimental instrument factory), ultrasonic grinder (JY92-II, Ningbo Kechuang Biotechnology Co., Ltd., China), constant temperature oscillator (DGHZ-300A, Beijing Dingguo Biotechnology Co., Ltd.), AFM (dimension icon, Bruce, USA), XPS (axis ultra DLD, Kratos analytical, UK), micro confocal Raman spectrometer (rts-hir-am, Vanguard technology Hong Kong Co., Ltd.), UV visible spectrophotometer (UV754N, Shanghai Precision Scientific Instrument Co., Ltd.), Fourier transform infrared spectrometer (FTIR-650, Tianjin Gangdong Technology Development Co., Ltd.), microelectronic printer (Scientific 3A, Shanghai Power Electronic Technology Co., Ltd.), Agilent digital source meter (B2902A, Shanghai Power Electronic Technology Co., Ltd.), Agilent Technologies Inc., USA.

\section{Results and discussion}

\subsection{Electronic features of the basic GFET}

The basic electronic features of the bare GFETs are examined, as shown in Fig. 1. In which, the output currents $\left(\mathrm{I}_{\mathrm{DS}}\right)$ vs. the voltage between the electrodes of source and drain $\left(\mathrm{V}_{\mathrm{DS}}\right)$, when the bias voltages between the electrodes of gate and source $\left(\mathrm{V}_{\mathrm{GS}}\right)$ were changed from -0.5 to $+0.5 \mathrm{~V}$, are plotted in Fig. 1 (A). It could be found there are small oxidation/reduction peaks, when $\mathrm{V}_{\mathrm{DS}}$ were located at about $\pm 0.3 \mathrm{~V}$, which are deduced to be caused by the redox of the oxygen containing groups on LEG. In the application of the DNA methylation detection, these redox reactions induced currents should be avoided, so in the following experiments the voltage of $\mathrm{V}_{\mathrm{DS}}$ is $0.5 \mathrm{~V}$. At this optimized working condition, the transfer characteristic curve was examined and shown in Fig. 1 (B), which is bipolar and in agreement with GFETs' typical features. It could be found the current at $\mathrm{V}_{\mathrm{GS}}=0.5 \mathrm{~V}$ is higher than the other bias voltages $\left(\mathrm{V}_{\mathrm{GS}}\right)$. Therefore, the bias voltages of $\mathrm{V}_{\mathrm{GS}}$ and $\mathrm{V}_{\mathrm{DS}}$ are controlled at $0.5 \mathrm{~V}$.
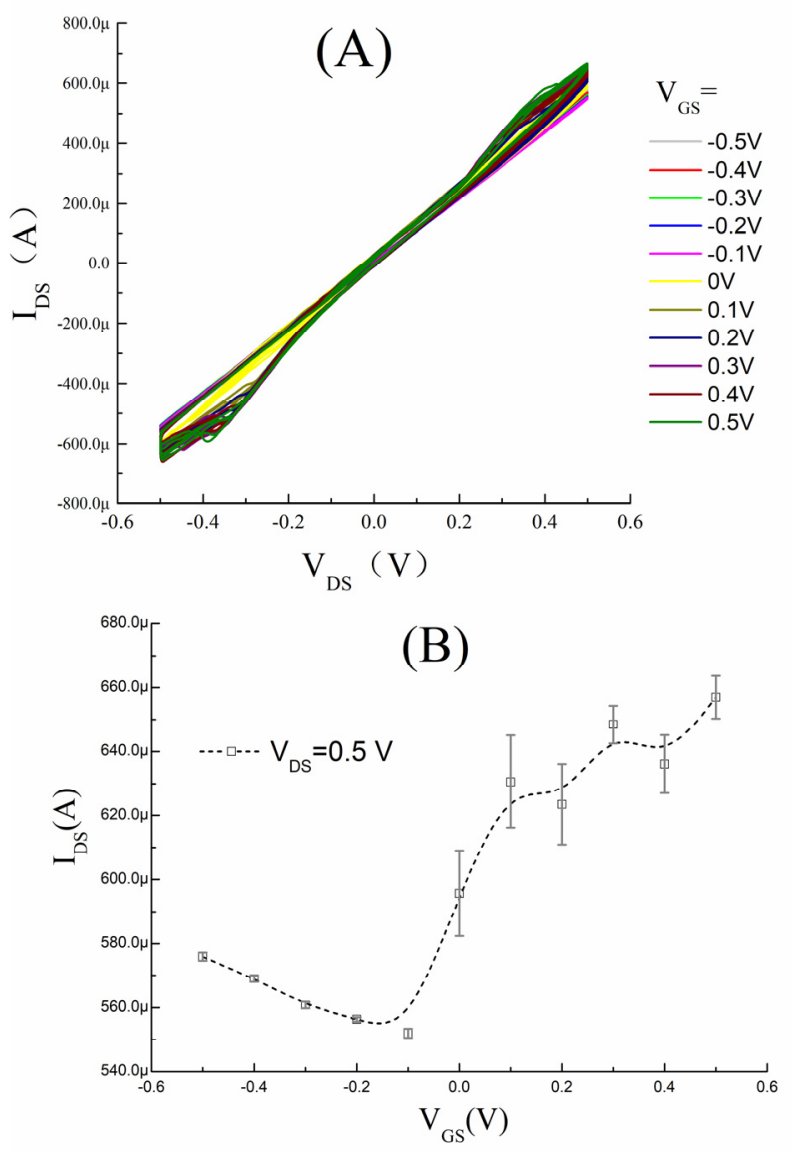

Fig. 1. The electronic features of the as-prepared LEG based GFET. (A) the output current (IDS) vs. voltage $\left(V_{D S}\right)$ curves at different bias voltage of $\mathrm{V}_{\mathrm{GS}}(-0.5$ to $0.5 \mathrm{~V})$. (B) transfer characteristic curve when $\mathrm{V}_{\mathrm{DS}}$ is $0.5 \mathrm{~V}$.

\subsection{CDs decoration}

For making sure the amount of CDs during the operation of CDs decoration, we measured $\mathrm{I}_{\mathrm{DS}}$ changing ratios after being modified by different CDs' concentrations $(0.0034,0.034,0.34,3.4,34,68,135 \mu \mathrm{g} / \mathrm{mL})$, as being plotted in Fig. 2. In which, $\mathrm{I}_{\mathrm{DS} 0}$ is the current before the CDs were coated, $\triangle I_{D S}$ is the variation of the current $I_{D S}$ minus $\mathrm{I}_{\mathrm{DS} 0}$, i.e., $\Delta \mathrm{I}_{\mathrm{DS}}=\mathrm{I}_{\mathrm{DS}}-\mathrm{I}_{\mathrm{DS} 0}$. It could be found more decreased currents could be measured with the increasing of CDs concentrations. We deduce it is caused by the increased impedance on the graphene 
channel, which may consume the voltage of $\mathrm{V}_{\mathrm{GS}}$, and lower the potential actually applied on the channel. Meanwhile, after the turning point (about $34 \mu \mathrm{g} / \mathrm{mL}$ ), the data are almost unchanged, it indicates the CDs on the channel of GFET is saturated though the exact amount cannot be known. So, $34 \mu \mathrm{g} / \mathrm{mL}$ CDs was used in the following experiments.

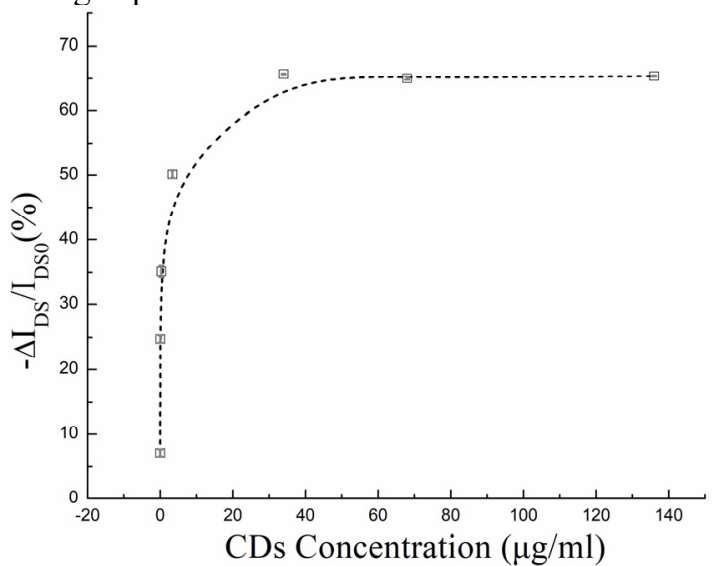

Fig. 2. The changed currents' ratio due to CDs' decoration.

\subsection{Immobilization of ssDNA}

After examining the above-mentioned basic electronic features, the tested ssDNA should be immobilized on the CDs decorated GFET in the third step. In this part, we need to ascertain what kind of influence might be induced by the anchored ssDNA. For this purpose, we measured the changed currents after the devices being incubated with different concentrations ssDNA, and plotted the data in Fig. 3. This changing tendency is similar to the CDs decoration induced current variations in Fig. 2. Likewise, with the increasing of concentrations, the current changing ratios tends to be saturated when the concentration of $\mathrm{T} 0$ is higher than $10 \mathrm{nM}$. We think it might be caused the increased impedance at the interface of liquid and graphene, which subsequently lower the potential actually applied on graphene.

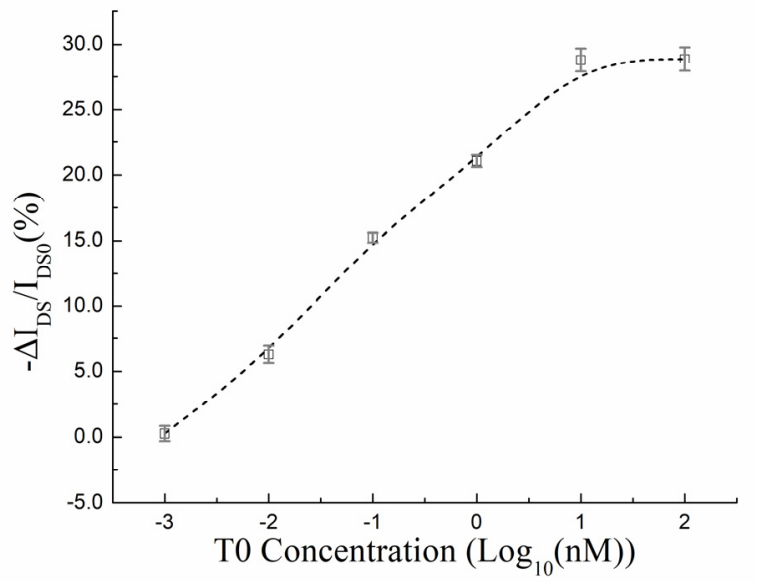

Fig. 3. The responding for the immobilized ssDNA (T0).

Moreover, because of the limitation of the surface sites to anchor ssDNA strands, the extra one cannot be fixed and no more changing could be measured when the concentration of ssDNA is $100 \mathrm{nM}$. According to this examination, the concentrations of the tested ssDNA in the next steps were controlled at $100 \mathrm{nM}$ to immobilize more ssDNA, as much as possible.

\section{$3.4 \mathrm{Immune}$ responding for anti-5mC}

In this work, the immune identification is utilized to distinguish the methylated "C" (i.e., $5 \mathrm{mC}$ ), so we need to make sure the current changing ratio is related to the captured molecules of anti-5mC. So, we examined the currents' variations after the T4 $(10 \mathrm{nM})$ anchored devices being incubated with the different concentrations of anti-5mC, as presented in Fig. 4. It could be found that the captured anti-5mC molecules might deduce the current and tend to be saturated at about $0.1 \mu \mathrm{g} / \mathrm{mL}$. This phenomenon means the $5 \mathrm{mC}$ sites on the anchored ssDNA are limited, the excessive anti-5mC molecules cannot be captured, or the influence of anti-5mC's nonspecific absorption can be neglected. In this examination, the tested ssDNA T4 is the one with the maximum $5 \mathrm{mC}$ sites, so we think the $0.1 \mu \mathrm{g} / \mathrm{mL}$ anti-5mC is enough for the other tested stands, and utilized for the following DNA methylation examinations.

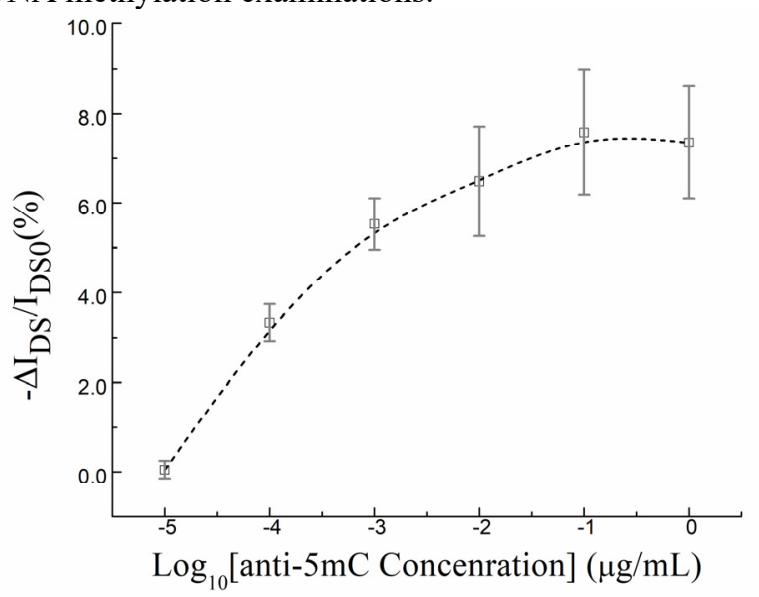

Fig. 4. The responding for the antibodies of $5-\mathrm{mC}$ (anti-5mC).

\subsection{DNA methylation examination}

To determine the amount $5 \mathrm{mC}$ on the tested ssDNA Ti $(i=0,1,2,3,4)$, we also need to record the currents' changing ratios and relate them with the amount of $5 \mathrm{mC}$ on the tested ssDNA, their sequences were artificially designed in Table 1. Since the only difference in them is the amount of $5 \mathrm{mC}$, we think it is reasonable to use them for evaluating the performance of proposed sensors for determining DNA methylation degree. The measured current changing ratios in relation with the amount of $5 \mathrm{mC}$ are plotted in Fig. 5. Since in this part, the abundant anti-5mC molecules were exerted on the $\mathrm{Ti}$ immobilized devices, and influence of the nonspecifically absorbed anti-5mC molecules is neglectable (as mentioned in the above section), we think the almost linearly changed data in relation with the increased amount of 5mC (as shown in Fig. 5) can proof the asprepared PET supported GFETs can be exploited as a DNA methylation sensor after being decorated by CDs and anchored the tested ssDNAs. 


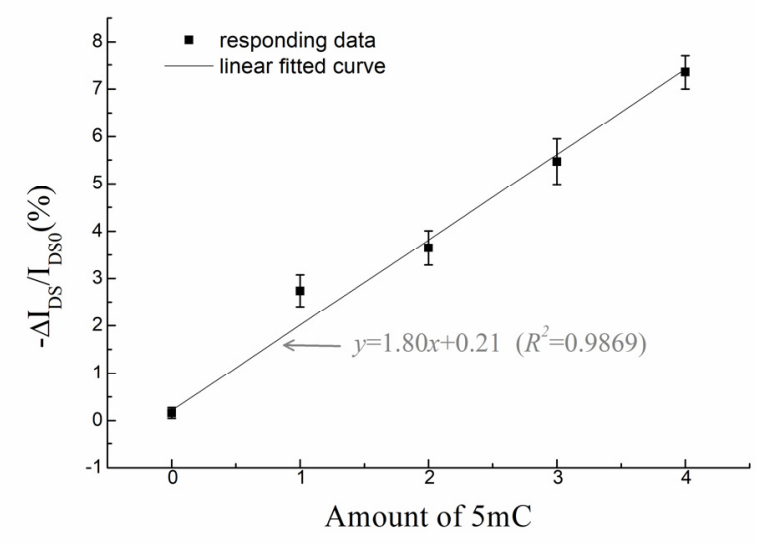

Fig. 5. The detection for the methylated ssDNA with different amount of $5 \mathrm{mC}$ positions.

\subsection{Interference examination}

To further investigate the interference coming from the un-methylated cytosine sites, we measured the devices' responding to the mixtures of $\mathrm{Ti}(\mathrm{i}=1,2,3,4)$ and $\mathrm{T} 0$ (named as Ti\&T0), with the volume ratio of $1: 1$, their concentrations are the same as in the section of 3.5 , i.e., $100 \mathrm{nM}$. The comparisons of the currents' responses to the mixtures of Ti\&T0 and the pure Ti are presented in Fig. 6. The halved columns of "Ti\&T0" were measured, this phenomenon can be explained as: all the ssDNA strands have similar chances to be anchored on the surface of GFET, the available sites for fixing ssDNA have be shared in the mixture of Ti\&T0, half-in-half. That is to say, both the un-methylated T0 strands and the methylated ones $\mathrm{Ti}(\mathrm{i}=1,2,3,4)$ can be anchored, therefore the currents responding is lowered about $50 \%$, contrasting to the pure Ti.

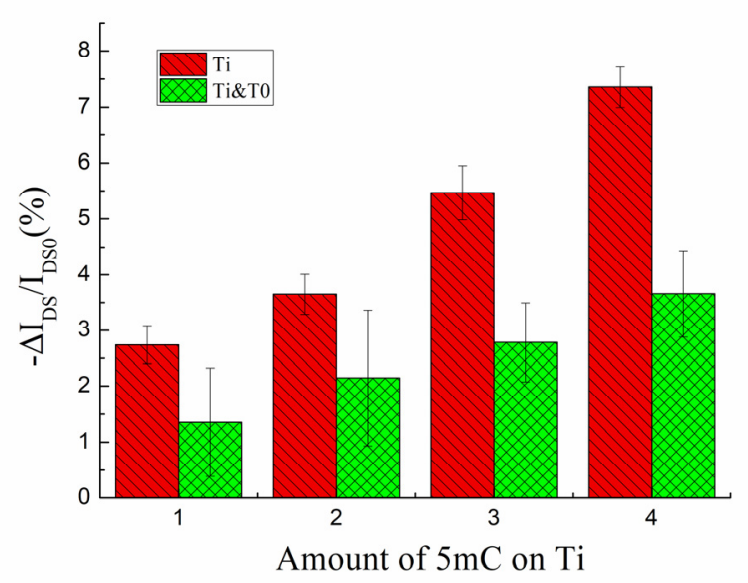

Fig. 6. The interference of un-methylated ssDNA.

\section{Conclusions}

In conclusion, we described and evaluated a method for developing the disposable DNA methylation sensors based on the GFET sensing platform. It is evidenced to have potential applicability to be exploited as an efficient, low-cost and easy-to-operate device for the epigenetic studies.

\section{Acknowledgments}

This work was supported by the National Natural Science Foundation of China [grant no. 61771260].

\section{References}

1. B. Chowdhury, I. H. Cho and J. Irudayaraj, J. Biol. Eng. 11, 10 (2017)

2. KC Kuo, A. McCune Roy, CW Gehrke, M Rosemarie, E Melanie, Nucleic Acids Res. 8(20), 4763 - 4776 (1980)

3. Z. Wang, S. Hu, F. Li, Q. Fan, Y. Jia, Analyst 144, 4787 - 4794 (2019)

4. M. Labib, E. H. Sargent, S. O. Kelley, Chem. Rev. 116(16), 9001, (2016)

5. D. Dong, J. Zhang, R. Zhang, F. Li, Y. Li, Y. Jia, ACS Omega 5, 16228 - 16237 (2020)

6. J. Ping, R. Vishnubhotla, A. Vrudhula, A. T. C. Johnson, ACS Nano 10(9), 8700 - 8704 (2016)

7. D. K. Ban, Y. Liu, Z. Wang, S. Ramachandran, R. Lal, ACS Nano 14(6), 6743 - 6751 (2020) 